\title{
Preparation, characterization, and evaluation of azoxystrobin nanosuspension produced by wet media milling
}

\author{
Junwei Yao ${ }^{1,2} \cdot$ Bo Cui $^{1} \cdot$ Xiang Zhao $^{1} \cdot$ Yan Wang ${ }^{1} \cdot$ Zhanghua Zeng $^{1} \cdot$ Changjiao Sun $^{1}$ - Dongsheng Yang ${ }^{1}$. \\ Guoqiang Liu ${ }^{1} \cdot$ Jinming $\mathrm{Gao}^{2} \cdot$ Haixin Cui $^{1}$
}

Received: 7 August 2017 / Accepted: 24 March 2018 / Published online: 18 April 2018

c) Springer-Verlag GmbH Germany, part of Springer Nature 2018

\begin{abstract}
To improve the bioavailability of the poorly water-soluble fungicide, an azoxystrobin nanosuspension was prepared by the wet media milling method. Due to their reduced mean particle size and polydispersity index, 1-Dodecanesulfonic acid sodium salt and polyvinylpyrrolidone K30 were selected from six conventional surfactants, the content only accounting for $15 \%$ of the active compound. The mean particle size, polydispersity index, and $\zeta$ potential of the nanosuspension were determined to be $238.1 \pm 1.5 \mathrm{~nm}, 0.17 \pm 0.02$ and $-31.8 \pm 0.3 \mathrm{mV}$, respectively. The lyophilized nanosuspension mainly retained crystalline state, with only a little amorphous content as determined by powder X-ray diffraction. Compared to conventional fungicide formulations, the nanosuspension presented an increased retention volume and a reduced contact angle, indicating enhanced wettability and adhesion. In addition, the azoxystrobin nanosuspension showed the highest antifungal activity, with a medial lethal concentration of $1.4243 \mu \mathrm{g} / \mathrm{mL}$ against Fusarium oxysporum. In optical micrographs, hyphal deformations of thinner and intertwined hyphae were detected in the exposed group. Compared to the control group, the total soluble protein content, superoxide dismutase, and catalase activities were initially increased and then decreased with prolonged exposure time. The azoxystrobin nanosuspension reduced the defensive antioxidant capability of Fusarium oxysporum and resulted in the generation of excessive reactive oxygen species. This study provides a novel method for preparing nanosuspension formulation of poorly soluble antifungal agents to enhance the biological activity and decrease the negative environmental impact.
\end{abstract}

Keywords Azoxystrobin · Nanosuspension · Antifungal activity $\cdot$ Fusarium oxysporum $\cdot$ Wet media milling

\section{Introduction}

Pesticides are vital to ensure modern agricultural harvests by controlling pests, diseases, and weeds. The problems of low bioavailability and extensive pesticide residues have caused the pollution of product, water and soil as well as the development of pesticide resistant (Bai et al. 2013; Radović et al. 2015; Castro et al. 2016). Currently, exceeding $40 \%$

Jinming Gao

jinminggao@nwsuaf.edu.cn

$\bowtie$ Haixin Cui

cuihaixin@caas.cn

1 Institute of Environment and Sustainable Development in Agriculture, Chinese Academy of Agricultural Sciences, Beijing 100081, China

2 Shaanxi Key Laboratory of Natural Products \& Chemical Biology, College of Chemistry \& Pharmacy, Northwest A\&F University, Yangling 712100, Shaanxi, China of commercially available drugs and $90 \%$ of novel active pharmaceutical components are extremely lipophilic/hydrophobic and exhibit poorly aqueous solubility (Krupa et al. 2016). As shown in Fig. 1, azoxystrobin, [methyl (E)-2-\{2[6-(2-cyanophenoxy)-pyrimidin-4-yloxy]phenyl \}-3-methoxyacrylate], is a strobilurin fungicide. A mitochondrial respiration inhibitor, azoxystrobin, is used to protect crops, vegetables, and fruits in the worldwide (Rodrigues et al. 2013; Kumari et al. 2015; Qin et al. 2016). Compounds with aqueous solubility less than $100 \mu \mathrm{g} / \mathrm{mL}$ have been defined as highly insoluble (Taylor and Zhang 2016). With an aqueous solubility of $6.7 \mu \mathrm{g} / \mathrm{mL}$, azoxystrobin has the characteristic of poorly soluble, typically resulting in a low bioavailability (Ghosh and Singh 2009; Kumari et al. 2015; Symonds et al. 2016). A new fungicide formulation with excellent aqueous solubility and low cost is essential for more effective utilization of azoxystrobin. Therefore, the enhancement of solubility is a challenging task in the development of azoxystrobin formulations. 
<smiles>CO/C=C(/C(=O)OC)c1ccccc1Oc1cc(Oc2ccccc2C#N)ncn1</smiles>

Fig. 1 The chemical structure of azoxystrobin

Basically, two approaches can be used to increase the solubility of a chemical component: physical techniques and chemical modifications (Mirza 2017). Physical techniques primarily include high-pressure homogenization, wet media milling, and carrier co-precipitation. In chemical modification, insoluble components are grafted with hydrophilic groups or transformed into salt forms. According to the Nernst-Brunner equation, a substance's solubility in water is negatively correlated with its particle size (Brough and Williams 2013). Hence, reducing the particle size is an effective approach to improve the solubility of hydrophobic compounds. With the development of nanotechnology, the application of nanomaterials has attracted wide attention in the field of agriculture, such as pesticide and fertilizer (Kah and Hofmann 2014). Recently, nanosuspensions with increased particle surface area have become one of the most promising formulations to enhance solubility (Yadollahi et al. 2015; Kumar Singh et al. 2016). Wet media milling has been regarded as a top-down approach for the industrial production of nanosuspensions and benefits from high efficiency, low cost, and free of organic residue (Ghosh et al. 2012; Li et al. 2016). Based on these superiorities, wet media milling provides a novel and easy method to produce poorly soluble fungicides.

Azoxystrobin inhibits mitochondrial electron transport in the respiratory chain as most strobilurin fungicides. The inhibitors accelerate electrons escaping from mitochondria, which is hastened by the generation of reactive oxygen species (ROS) (Turrens and Boveris 1980; Olsvik et al. 2010). The fungi form a set of antioxidant defense system inclusive of superoxide dismutase (SOD) and catalase (CAT) (Azevedo et al. 2007). However, excess ROS at the early stages of mitochondrial disruption can lead to fungi death (Inoue et al. 2011).

In this study, an azoxystrobin nanosuspension was prepared by wet media milling. The particle size and $\zeta$ potential of the nanosuspension were measured by dynamic light scattering (DLS). The morphology and structure of the nanoparticles were characterized by scanning electron microscopy (SEM) and transmission electron microscopy (TEM). The contact angle and retention volume on the leaves of cucumbers and cabbages were measured. Compared with commercially available formulations, antifungal activity of the nanosuspension was tested by potato dextrose agar (PDA) assay. The morphologies of Fusarium oxysporum were observed by optic microscopy and SEM. This study also evaluated the effects of the azoxystrobin nanosuspension on fungal protein content, SOD, and CAT.

\section{Experimental section}

\section{Materials}

Azoxystrobin (97\%) was purchased from Hubei Sheng Tianheng record Biological Technology Co., Ltd. (Hubei, China). 1-Dodecanesulfonic acid sodium salt (SDS), polyvinylpyrrolidone K30 (PVP K30), hexadecyl trimethyl ammonium chloride (CTAC), and polyoxyethylene sorbitan monooleate (Tween 80 ) were provided by J\&K Scientific Ltd. (Beijing, China). Poloxamer 188 (F68) was obtained from SigmaAldrich (Shanghai, China). Polycarboxylate was provided as a gift by Sinvochem S\&D Co., Ltd. (Jiangsu, China). Commercially available water dispersible granules (WDG) were purchased from Jiangsu KWIN Group (WDG-A) and Jiangsu Huifeng Agrochemical Co., Ltd. (WDG-B). HPLCgrade methanol was obtained from Thermal Fisher Scientific (Tustin, CA, USA). Deionized Milli-Q water was used in all experiments $(18.2 \mathrm{M} \Omega \cdot \mathrm{cm}, \mathrm{TOC} \leq 4 \mathrm{ppb})$.

\section{Methods}

\section{Preparation of azoxystrobin nanosuspension}

Azoxystrobin nanosuspension was manufactured by wet media milling. In brief, azoxystrobin powder was dispersed in an aqueous solution containing one of the six surfactants (SDS, PVP K30, F68, Tween 80, CTAC, and polycarboxylate), and the solution was subjected to mechanical stirring (Ika, mod. RW 20 digital, Germany) at $1000 \mathrm{rpm}$ for $30 \mathrm{~min}$. The suspension (6\% azoxystrobin) was then milled with the milling media apparatus (WG-0.3 L, Vgreen nano-tech, China). The grinding chamber $(0.3 \mathrm{~L})$ was made of silicon carbide, and $80 \%$ of the chamber was filled with $0.3-\mathrm{mm}$ zirconium oxide beads as the milling medium. The azoxystrobin crystals were fragmented into nanoparticles by the physical impact of the zirconium oxide beads at a speed of $2200 \mathrm{rpm}$.

\section{Particle size and $\zeta$ potential determination of the nanosuspension}

The mean particle size, polydispersity index (PDI), and $\zeta$ potential of the nanosuspension were analyzed by DLS with a Zetasizer Nano ZS90 (Malvern Instruments, UK) at 
room temperature. Each sample was measured three times for reliability.

\section{Morphological characterization of the nanoparticles by SEM and TEM}

The morphology of the azoxystrobin nanosuspension was evaluated using SEM (SU8010, Hitachi, Tokyo, Japan) at an acceleration voltage of $5 \mathrm{kV}$. The sample was placed on a clean silicon slice, dried at room temperature, and then sputtered with platinum under vacuum (EM ACE600, Leica, Germany). The size and morphology of the nanosuspension were characterized by TEM (HT7700, Hitachi, Tokyo, Japan) at an operational voltage of $80 \mathrm{kV}$. The sample was dropped on carbon-coated 300-mesh copper grids.

\section{Powder X-ray diffraction analysis of the nanoparticles}

For X-ray diffraction analysis, the water of azoxystrobin nanosuspension was removed by lyophilization (FD-81, EYELA, Tokyo, Japan). The patterns of samples were analyzed by a diffractometer (D8 ADVANCE, Bruker AXS Inc., Karlsruhe, Germany) with a $\mathrm{Cu} \mathrm{K} \alpha$ radiation source, operated at $40-\mathrm{kV}$ voltage and $40-\mathrm{mA}$ current. Scans were recorded with a detector rate of $0.2^{\circ} / \mathrm{min}$ and $2 \theta$ range from 5 to $50^{\circ}$.

\section{Wetting and spreading characteristics}

Hydrophobic leaf of cabbage and hydrophilic leaf of cucumber were cultivated by light growth incubator. The contact angles of the droplets were measured with a contact angle apparatus (JC2000D2 M, Zhongchen Digital Technology Apparatus, Shanghai, China). The $7-\mu \mathrm{L}$ diluted suspension was dropped onto the leaf using a $50-\mu \mathrm{L}$ syringe.

The retention of the sample on the leaf was determined by the dipping method. A $15-\mathrm{mm}$-diameter leaf was perforated by a hole punch and weighed as $\mathrm{M}_{1}$. The leaf was immersed in the diluted suspension for $10 \mathrm{~s}$. Until no more droplets falling, the leaf was removed and weighed as $\mathrm{M}_{2}$. The measurements were conducted at room temperature. Five tests were performed for each sample.

\section{Azoxystrobin concentration analysis}

The azoxystrobin concentration was estimated by highperformance liquid chromatography (HPLC) (Agilent 1260 series HPLC, Agilent Technologies) using a Zorbax Carbohydrate Analysis column $(150 \mathrm{~mm} \times 4.6 \mathrm{~mm} \times 5 \mu \mathrm{m})$ and a 254-nm UV detector. The mobile phase consisted of methanol and water $(75: 25, \mathrm{v} / \mathrm{v})$ at a flow rate of $0.8 \mathrm{~mL} / \mathrm{min}$.

\section{In vitro dissolution}

The suspensions commensurate with effective azoxystrobin were put into dialysis bags (2000 MWCO). The bags were then suspended in $100 \mathrm{~mL}$ of an ethanol/deionized water solution $(50: 50, \mathrm{v} / \mathrm{v})$ as the release medium. The solution was shaken at $100 \mathrm{rpm}$ at $28{ }^{\circ} \mathrm{C}$ using a constant temperature table (THZ-98C, Shanghai, China). A $2 \mathrm{~mL}$ of the outside release medium was removed at different timed intervals. Meanwhile, $2 \mathrm{~mL}$ of fresh mix solution was added back into the sustained release system. For quantitative analysis, the azoxystrobin concentration in the $2 \mathrm{~mL}$ of release medium was determined by HPLC.

\section{Fungal antagonism assays}

To examine fungal antagonism, Fusarium oxysporum was used for the antifungal activity test with PDA assay. Various azoxystrobin formulations containing the nanosuspension, WDG-A or WDG-B were precisely prepared with the following concentrations: $0.1,0.25,0.5,1.0$ and $5.0 \mu \mathrm{g} / \mathrm{mL}$. A 5 -mm-diameter mycelial disc was incubated on the test medium at $28 \pm 1{ }^{\circ} \mathrm{C}$ for 2 days. The diameter of mycelium growth was determined by the criss-cross method. The toxicity regression equations and the medial lethal concentration $\left(\mathrm{LC}_{50}\right)$ were calculated by probit analysis using SPSS 20 statistical software (IBM Corp., Armonk, NY, USA). Each experiment was implemented in triplicate.

\section{Fungal hyphae microscopy analysis}

Fusarium oxysporum was cultured with PDA, and then a 10-mm-diameter mycelial disc was incubated in the potato dextrose broth (PDB) medium at $28 \pm 1{ }^{\circ} \mathrm{C}$ for 2 days. To evaluate the effect of the azoxystrobin nanosuspension on Fusarium oxysporum, the azoxystrobin nanosuspension with a final concentration of $5.0 \mu \mathrm{g} / \mathrm{mL}$ was added to the PDB medium. The hyphae from control and azoxystrobin-treated groups were collected after $24 \mathrm{~h}$. Bright field microscopy images were observed using an inverted fluorescence microscope (Olympus IX71, Tokyo, Japan) with a 40-time objective lens. Furthermore, the hyphae were fixed with a $2.5 \%$ glutaraldehyde solution at $4{ }^{\circ} \mathrm{C}$ overnight. The fixed hyphae were washed with phosphate-buffered saline (PBS) solution ( $\mathrm{pH}$ 7.4) three times for $10 \mathrm{~min}$. Afterward, the washed hyphae were dehydrated with 50,70, 90, and $100 \%$ ethanol for $10 \mathrm{~min}$ and then were further dehydrated with absolute ethanol for $20 \mathrm{~min}$. The dehydrated hyphae were dropped onto clean silicon slices and sputtered with platinum under vacuum (EM ACE600, Leica, Germany). The morphology

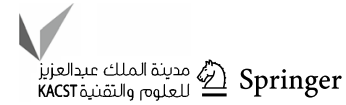


of the hyphae was recorded with SEM (SU8010, Hitachi, Tokyo, Japan) at an acceleration voltage of $5 \mathrm{kV}$.

\section{SOD and CAT assays}

The Fusarium oxysporum exposed to azoxystrobin nanosuspension $(5 \mu \mathrm{g} / \mathrm{mL})$ was collected to extract protein at different times after exposure for 1, 6, 18, 24, and $48 \mathrm{~h}$, respectively. Protein was extracted by milling hyphae with a glass homogenizer in an ice bath. The homogenate was centrifuged (SORVALL ST16R, Thermo Scientific, MA, USA) at $10,000 \mathrm{rpm}$ for $20 \mathrm{~min}$ at $4{ }^{\circ} \mathrm{C}$. The supernatant was collected and used for the analysis of antioxidant enzyme activities. The concentration of soluble protein was measured by the Bradford method (1976) using bovine serum albumin (BSA) as a standard protein. SOD and CAT activities were determined by UV spectrophotometry (UV-2600, Shimadzu, Japan) using commercial assay kits (Jiancheng Institute, Nanjing, China).

\section{Statistical analysis}

Data analysis was performed using SPSS 20 statistical software (IBM Corp., Armonk, NY, USA). Data were presented as the mean \pm standard deviation (SD). Quantitative data of contact angle and retention volume were evaluated with a one-way analysis of variance (ANOVA) followed by the least significant difference (LSD) method. A probability less

Table 1 The effect of surfactant on the mean particle size and PDI of the nanosuspension

\begin{tabular}{lcl}
\hline Surfactant & Mean size $($ d.nm $)$ & PDI \\
\hline Tween 80 & $2054.0 \pm 216.20$ & $0.52 \pm 0.07$ \\
F68 & $1233.3 \pm 39.11$ & $0.59 \pm 0.07$ \\
SDS & $307.5 \pm 5.09$ & $0.18 \pm 0.01$ \\
Polycarboxylate & $854.7 \pm 60.25$ & $0.45 \pm 0.07$ \\
PVP K30 & $344.9 \pm 5.39$ & $0.07 \pm 0.02$ \\
CTAC & $661.0 \pm 8.63$ & $0.16 \pm 0.03$ \\
\hline
\end{tabular}

than 0.05 was considered statistically significant. The other quantitative data were examined using a one-way ANOVA followed by the Student-Newman-Keuls (SNK) method.

\section{Results and discussion}

\section{Preparation of the azoxystrobin nanosuspension}

The influence of six surfactants on the nanosuspension was examined using mean particle size and PDI as measuring indices. Nanosuspensions containing 6\% (w/w) azoxystrobin and $0.9 \%$ surfactant $(\mathrm{w} / \mathrm{w})$ were prepared by wet media milling at $2200 \mathrm{rpm}$ for $20 \mathrm{~min}$. As shown in Table 1, surfactants had significant effects on the mean particle size and the dispersity of the suspension. Among the six investigated surfactants, SDS and PVP K30 were screened out, which could reduce the mean particle size of the suspension less than $400 \mathrm{~nm}$ and PDI no more than 0.25. A PDI less than 0.25 suggested that the suspension had a narrow distribution (Ibrahim et al. 2014; Ninjbadgar et al. 2015). During the milling process, nanoparticle agglomeration was mainly caused by van der Waals forces and electrostatic interactions (Rance et al. 2010; Moerz and Huber 2015). For anionic surfactant, SDS could provide sufficient electric charge to induce electrostatic repulsion for the suspended particles (Teeranachaideekul et al. 2008). For covering the surface of the particles, nonionic surfactant PVP K30 could prevent nanosuspensions from aggregating by the steric hindrance effect (Palermo et al. 2012). Appropriate surfactants were crucial to inhibit particle agglomeration and to keep the system stable (Elsayed et al. 2014). To combine electrostatic repulsion and steric stabilization, SDS and PVP K30 $(1: 1, w / w)$ were mixed to prepare the azoxystrobin nanosuspension. As shown in Fig. 2, particle size and PDI did not decrease further with extended grinding time (George and Ghosh 2013). In contrast, a wet grinding time of more than 90 min caused the particle size to increase and the PDI to broaden. A potential reason for this effect was that high shear mechanical force could lead to secondary aggregation
Fig. 2 The effect of milling time on the characteristics of the nanosuspension. (a) mean particle size; (b) PDI
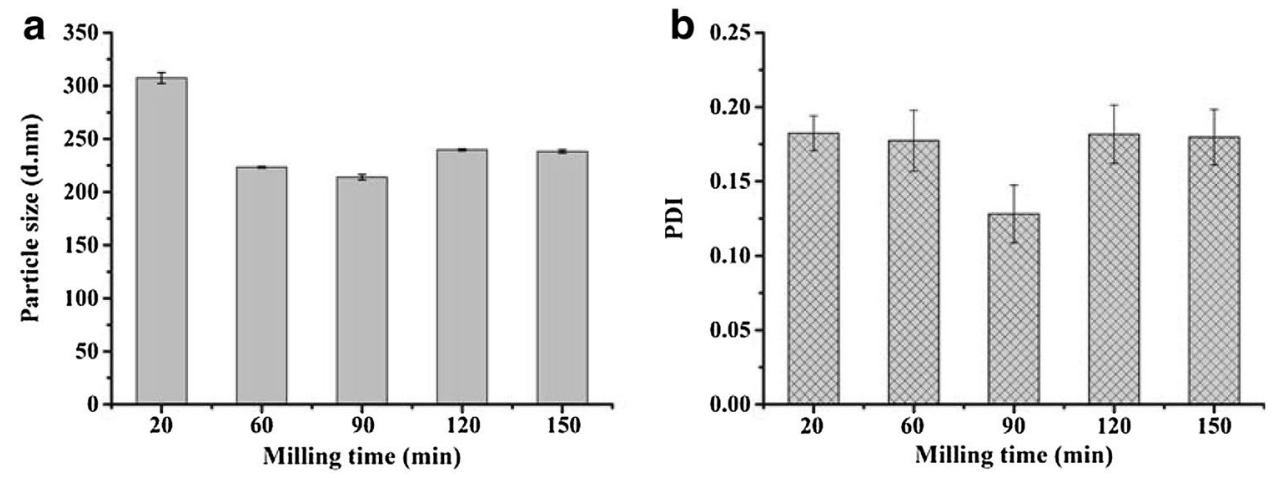
during milling, depending on the temperature (Kargosha and Shirazi 2015).

\section{The particle size and $\zeta$ potential}

The mean particle size, $\mathrm{D}_{90}$ and PDI of the aqueous diluted nanosuspension measured by DLS were $238.1 \pm 1.5 \mathrm{~nm}$, $463.7 \pm 17.7 \mathrm{~nm}$, and $0.17 \pm 0.02$, respectively. A suspension with an absolute $\zeta$ potential higher than $30 \mathrm{mV}$ implicated a stable system by electrostatic repulsion (Pamies et al. 2014). As shown in Fig. 3b, the $\zeta$ potential of the aqueous diluted nanosuspension was $-31.8 \pm 0.3 \mathrm{mV}$. The highly negative $\zeta$ potential value can be attributed to anionic SDS and indicates excellent stability.

\section{Morphology}

To evaluate the shape of the nanoparticles, samples were observed by SEM and TEM. Based on Fig. 4a, c, the particles exhibited irregular bulk shape. The sizes of particles were from 80 to $530 \mathrm{~nm}$, with an average diameter of around $200 \mathrm{~nm}$, which was approximately $16 \%$ smaller than that measured by DLS. The reason may lie in the disparate particle morphology (Cho et al. 2004). Electron microscopy images showed the veritable size of a single particle in a dried state (Mcallister et al. 2016). In contrast, the size measured by dynamic light scattering technique was a hydrated state value of spherical particles, reflecting a single particle or aggregates (Zhong and Jin 2009; Singh et al. 2017).
Fig. 3 The characteristic of nanosuspension by DLS. a hydrodynamic particle size; $\mathbf{b} \zeta$ potential
Fig. 4 The morphologies of the nanosuspension characterized by electron microscopy. a SEM image; b statistical particle size based on SEM; $\mathbf{c}$ TEM image; d statistical particle size based on TEM
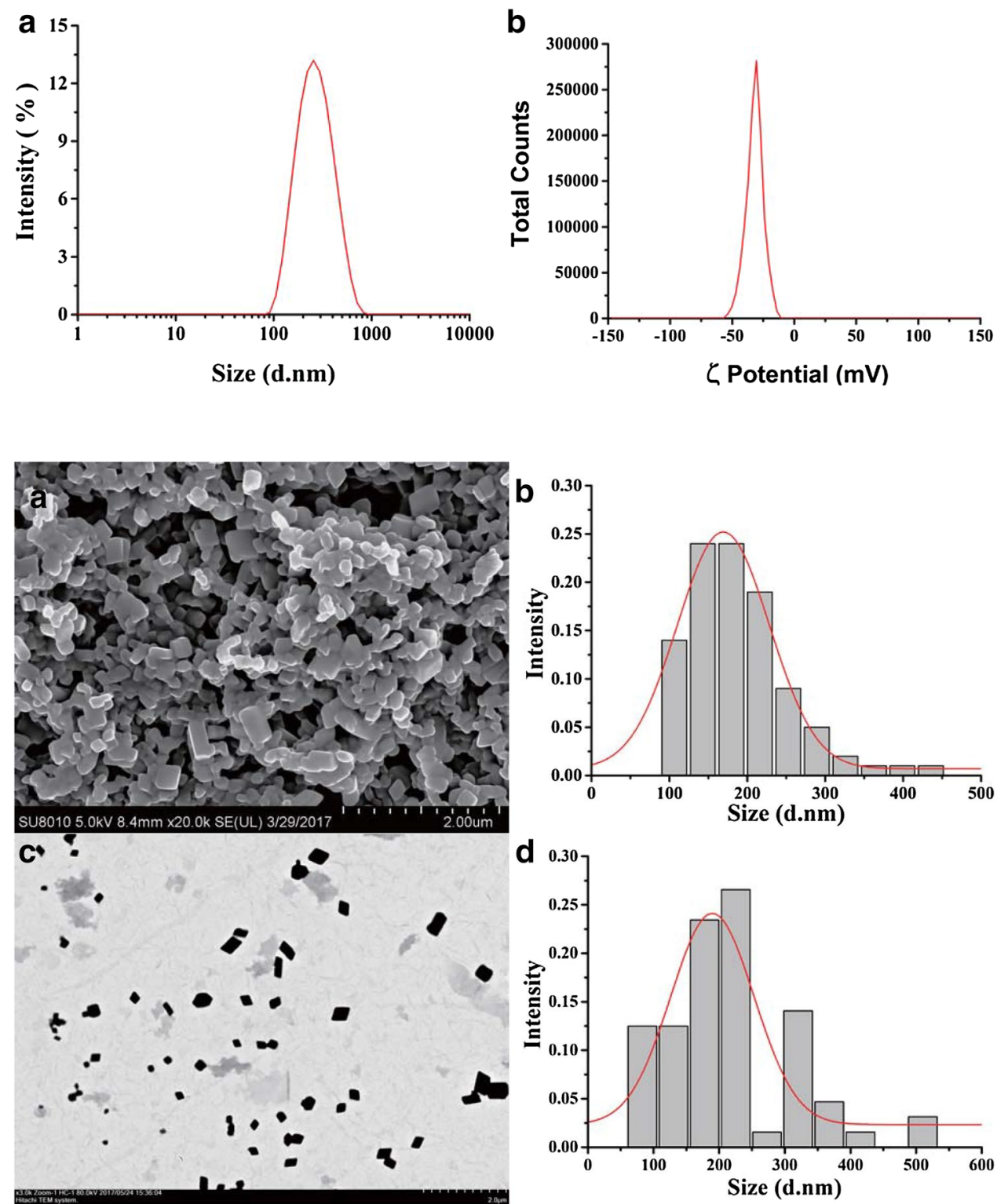


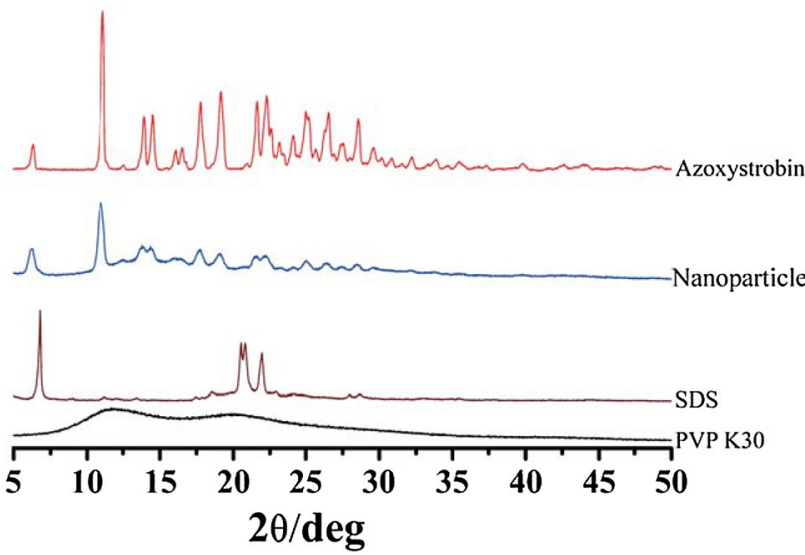

Fig. 5 XRD patterns of lyophilized azoxystrobin nanoparticles and related ingredients

\section{Crystalline state}

As shown in the X-ray diffraction spectrum (Fig. 5), the characteristic peaks of lyophilized azoxystrobin nanoparticles were at 11.0, 13.8, 17.7, 19.1 and $22.2^{\circ}$. The characteristic diffraction peaks of the nanoparticles could be primarily attributed to azoxystrobin as it accounted for $80 \%$ of the ingredients in the lyophilized powders. As previously reported, the wet milling process induced violent collisions between the milling media and the substance, which can destroy or disorder the crystal structure (Lee et al. 2015). During the grinding period, the high rate of rotation of the zirconium oxide media could bring out massive heat and mechanical shearing force, which probably enhanced lattice vibrations and changed the crystalline state into an amorphous one (George and Ghosh 2013; Ito et al. 2016). In other words, wet media milling may cause changes in the physical structure of the organic components such as crystal defects or crystalline transition. XRD analysis revealed that lyophilized nanoparticles principally maintained a crystalline state, only a little of amorphous content.

\section{In vitro dissolution of various azoxystrobin formulations}

The release kinetics of azoxystrobin in different formulations were shown in Fig. 6. After fitting the accumulated release data with multiple kinetic models, the results demonstrated that the release kinetic profiles were well corresponding with the first-order kinetics, with $R^{2}$ value over 0.99 . As seen from the accumulated release trend, $50 \%$ release from the nanosuspension, WDG-A and WDG-B was observed at $0.99,3.93$, and $4.33 \mathrm{~h}$, respectively. In previous research describing the impact of nanosizing on dissolution rates, griseofulvin nanocrystals took less than 2 min to release $80 \%$

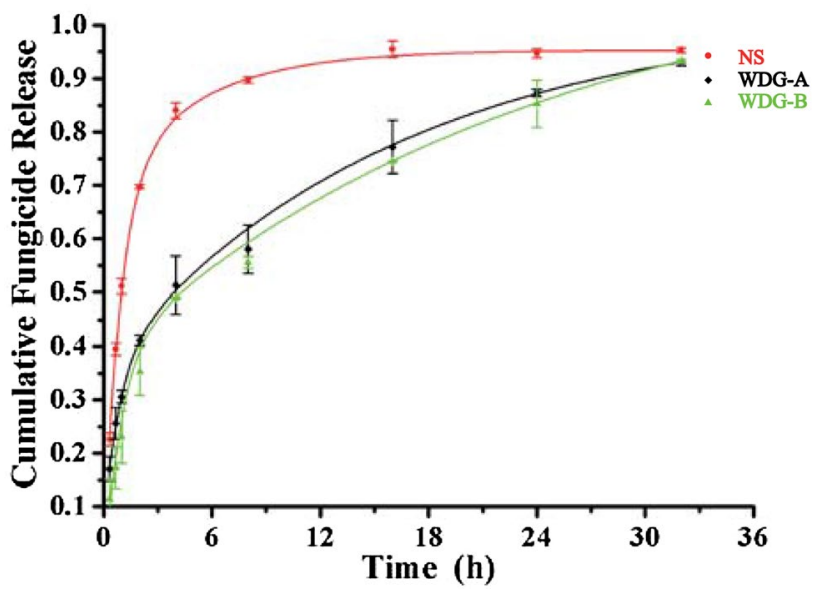

Fig. 6 Cumulative release profiles of azoxystrobin derived from nanosuspension (NS), WDG-A and WDG-B

of the nanocrystals, while a micronized suspension required more than 20 min (Murdande et al. 2015). A similar phenomenon was previously observed using paliperidone palmitate nanosuspensions with various particle size (Leng et al. 2014). Likewise, the azoxystrobin nanosuspension showed an enhancing dissolution rate compared to the commercial WDG formulation. Based on the Ostwald-Freundlich equation, particle size had a significant effect on the dissolution rate of poorly soluble compounds (Murdande et al. 2015). Furthermore, increased surface area most likely improved dissolution rates according to the Noyes-Whitney equation. The improved dissolution rate of the azoxystrobin nanosuspension was a result of the substantially reduced particle size.

\section{Stability of the nanosuspension}

As crucial indices to evaluate stability, the particle size and distribution of the nanosuspension were determined by DLS (Ali et al. 2009). To estimate the effect of temperature on azoxystrobin nanosuspension stability, the mean particle size and distribution changes were recorded after storage at 4,25 and $54{ }^{\circ} \mathrm{C}$, respectively. As shown in Fig. 7a, the mean particle size of the nanosuspension increased from 238 to $288 \mathrm{~nm}$ over 14 days of storage at $25{ }^{\circ} \mathrm{C}$. As seen from Fig. 7b, a similar particle size variation was observed at $4{ }^{\circ} \mathrm{C}$ for 7 days. The initial mean particle size increased by $30 \%$ during storage for 14 days at $54{ }^{\circ} \mathrm{C}$ (Fig. $7 \mathrm{c}$ ). A reasonable explanation was that nanoparticle agglomeration likely occurred during high-temperature storage (Yue et al. 2015). PDI reflected the distribution width of particle size and was another reference index to characterize suspension stability (Holzapfel et al. 2005). The PDIs of the nanosuspensions stored at different temperatures were all less than 0.25 , indicating a narrow particle distribution (Fig. 7). 

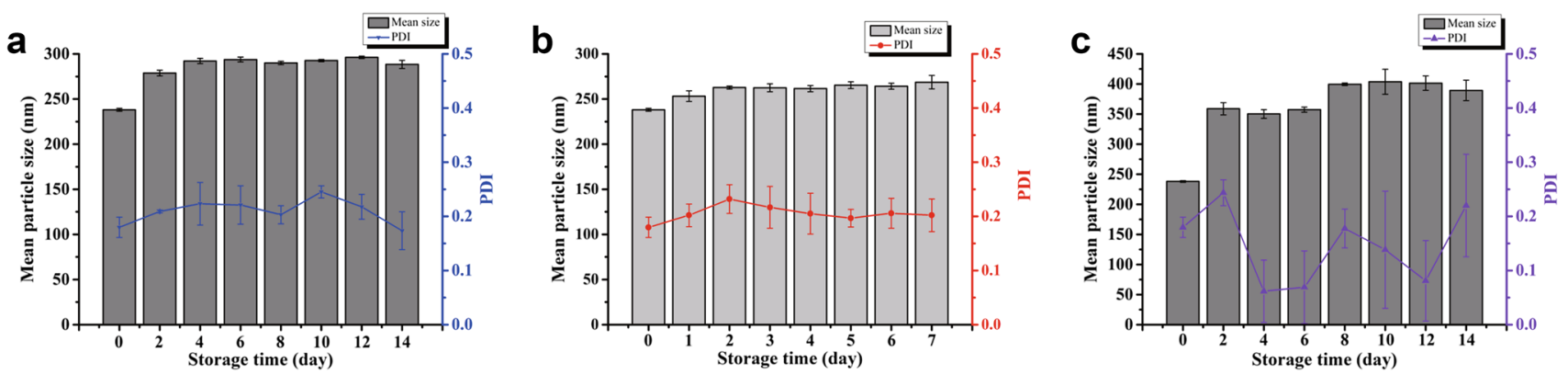

Fig. 7 The effect of temperature on particle size and PDI. a $25{ }^{\circ} \mathrm{C}$; b $4{ }^{\circ} \mathrm{C}$; c $54{ }^{\circ} \mathrm{C}$

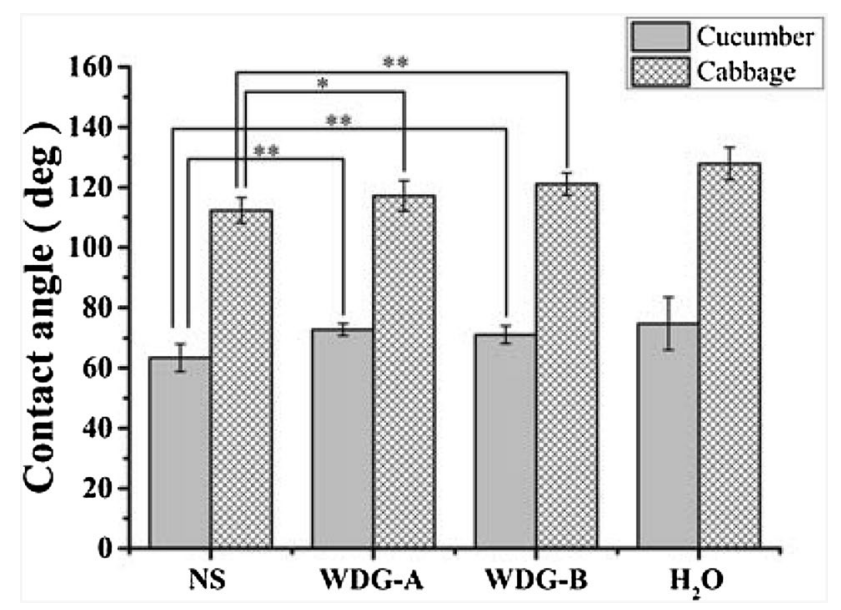

Fig. 8 The contact angles of azoxystrobin formulations on cucumber and cabbage surfaces (one-way ANOVA, followed by LSD test, $* p<0.05, * * p<0.01$ )

\section{Wetting and spreading characteristics}

To evaluate the wettability behavior of the nanosuspension on cucumber and cabbage leaves, contact angles and retention volumes were measured. The contact angle was a vital criterion to assess the hydrophilic and hydrophobic properties of suspensions (Sun et al. 2016). As shown in Fig. 8, the contact angle of water on the cucumber leaf surface was $75^{\circ}$ (less than $90^{\circ}$ ), reflecting the hydrophilic characteristic of cucumber leaf (Lin et al. 2016). On the contrary, the contact angle of water on a cabbage leaf was $128^{\circ}$, implying a hydrophobic characteristic. On cucumber leaves, the contact angles of the nanosuspension, WDG-A, and WDG-B were $63^{\circ} \pm 5^{\circ}, 73^{\circ} \pm 2^{\circ}$, and $71^{\circ} \pm 3^{\circ}$, respectively. Meanwhile, the contact angles on cabbage surfaces were $112^{\circ} \pm 4^{\circ}$, $117^{\circ} \pm 5^{\circ}$, and $121^{\circ} \pm 4^{\circ}$, respectively. The smaller contact angle of the droplet indicated that the liquid was easier to spread and wet on the leaf surface (Zeng et al. 2015). Significantly different contact angles were observed when comparing the nanosuspension to WDG-A and WDG-B. The azoxystrobin nanosuspension possessed smaller contact

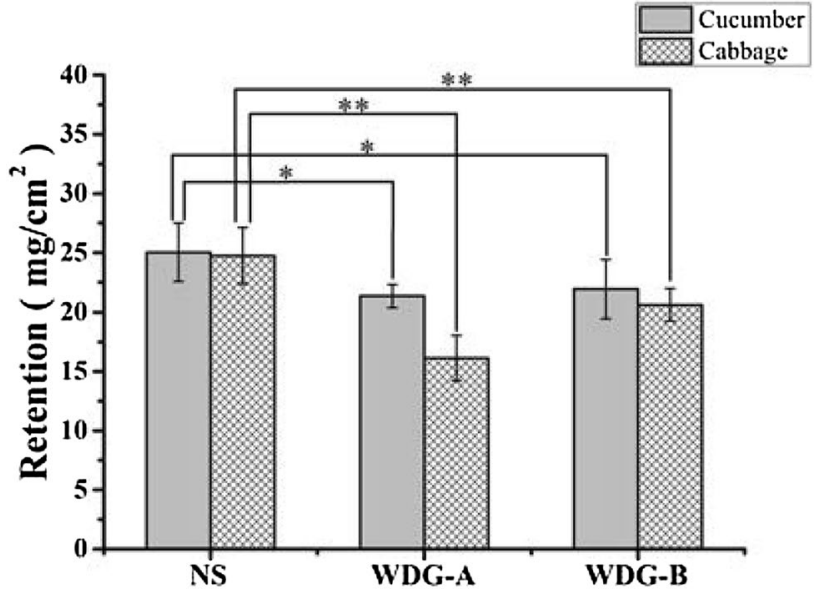

Fig. 9 The retention volumes of azoxystrobin formulations on cucumber and cabbage surfaces (one-way ANOVA, followed by LSD test, $* p<0.05,{ }^{* *} p<0.01$ )

angles, which led to an enhancing wettability and spreading performance on cucumber and cabbage leaves. Retention volumes on cucumber and cabbage leaves were presented in Fig. 9. On cucumber leaves, the retention volumes of nanosuspension, WDG-A, and WDG-B were $25.04 \pm 2.47$, $21.34 \pm 0.95$ and $21.93 \pm 2.48 \mathrm{mg} / \mathrm{cm}^{2}$, respectively. On cabbage leaves, the retention volumes of nanosuspension, WDG-A, and WDG-B were $24.75 \pm 2.39,16.12 \pm 1.93$ and $20.63 \pm 1.36 \mathrm{mg} / \mathrm{cm}^{2}$, respectively. The retention volumes of the nanosuspension on cucumber and cabbage surfaces were higher than the commercially available WDG formulation, which may bring about the reduction in the pesticide dosage.

\section{Antifungal activity evaluation}

In this study, the PDA assay was evaluated the antifungal activity of three azoxystrobin formulations. The $\mathrm{LC}_{50}$ values were used as a measurement antifungal activity of azoxystrobin against Fusarium oxysporum. As shown in Table 2, the azoxystrobin nanosuspension exhibited the highest toxicity with an $\mathrm{LC}_{50}$ of $1.4243 \mu \mathrm{g} / \mathrm{mL}$, followed by WDG-A 
and WDG-B with $\mathrm{LC}_{50}$ values of 2.4668 and $2.4221 \mu \mathrm{g} / \mathrm{mL}$, respectively. The lower $\mathrm{LC}_{50}$ value of the azoxystrobin nanosuspension showed that its toxicity index was around 1.7fold that of the others. As reported, the antifungal activity of azoxystrobin was attributed to its ability to bind the $Q_{0}$ site of complex III in the fungus mitochondria, which resulted in the inhibition of mitochondrial respiration and blocked the synthesis of ATP (Gao et al. 2002). It was well known that the small size of nanoparticles increases their ability to penetrate cell membranes, giving them access to their target site (Verma et al. 2008). In conclusion, the antifungal activity of the azoxystrobin nanosuspension was superior to the conventional WDG formulations.

\section{Effect on mycelial morphology}

The Fusarium oxysporum optical morphology is illustrated in Fig. 10. Figure 10a reflected the control group, which maintained intact, chiseled septa, and a strong budding reproductive capacity. In contrast, Fig. 10b displayed that the hyphae tended to intertwine when exposed to the azoxystrobin nanosuspension. In comparison to the control group, the azoxystrobin nanosuspension caused deformation in the fungi, with the hyphae being thinner and the septa being poorly defined. A similar phenomenon was previously presented in the E. salmonicolor with different concentrations of $\mathrm{ZnO}$ nanoparticles (Arciniegas-Grijalba et al. 2017). The Fusarium oxysporum microstructure was shown in Fig. 11. Figure 11a displayed normal mycelia with smooth walls and intact surfaces without shrinkage. However, the mycelia treated with the azoxystrobin nanosuspension became severely shrunk and crumpled (Fig. 11b). In a previous study, Chlorella vulgaris cells were also apparently depressed or shrunk in 300 or $600 \mu \mathrm{g} / \mathrm{L}$ azoxystrobin treatments (Liu et al. 2015). When the azoxystrobin nanosuspension penetrated the Fusarium oxysporum cell wall and impacted on the mitochondria target site, it can disturb the cell wall and enhance its permeability. From the mycelial morphological examinations, azoxystrobin nanosuspension indeed inhibited the growth of Fusarium oxysporum.
Table 2 Indoor toxicity of three azoxystrobin formulations against Fusarium oxysporum

\begin{tabular}{lllll}
\hline Formulation & Regressive equation & $R^{2}$ & $\mathrm{LC}_{50}(\mu \mathrm{g} / \mathrm{mL})$ & Toxicity index \\
\hline Nanosuspension & $y=5.4764+0.1929 \mathrm{x}$ & 0.9971 & 1.4243 & 1.7319 \\
WDG-A & $y=5.4025+0.2440 \mathrm{x}$ & 0.9937 & 2.4668 & 1 \\
WDG-B & $y=5.4433+0.1465 \mathrm{x}$ & 0.9993 & 2.4221 & 1.0185 \\
\hline
\end{tabular}

Fig. 10 Optical microscopy images of Fusarium oxysporum hyphal structures. a control; b treatment with azoxystrobin nanosuspension
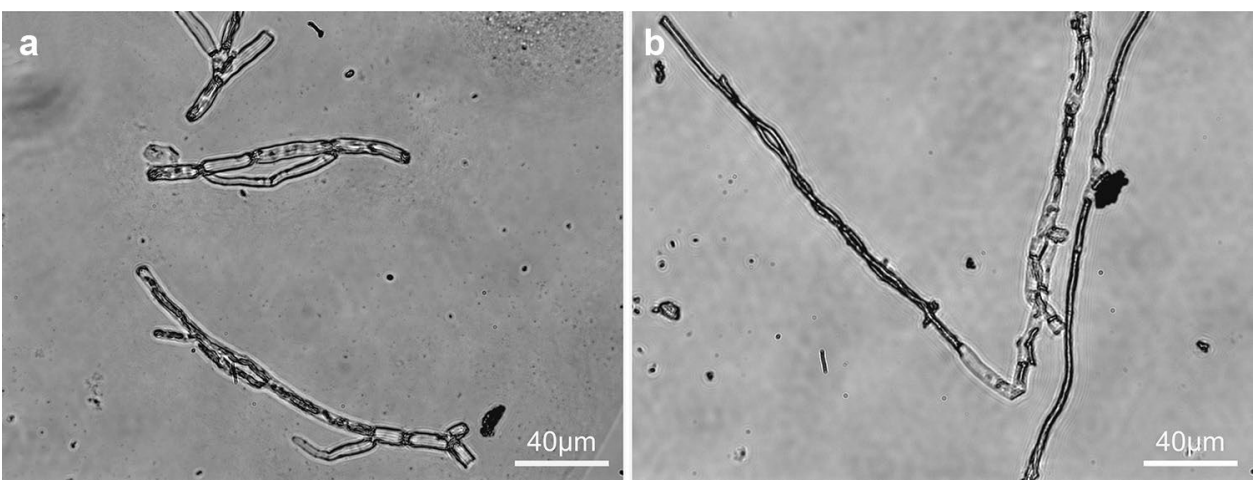

Fig. 11 SEM images of Fusarium oxysporum hyphal structures. a control; b treatment with azoxystrobin nanosuspension
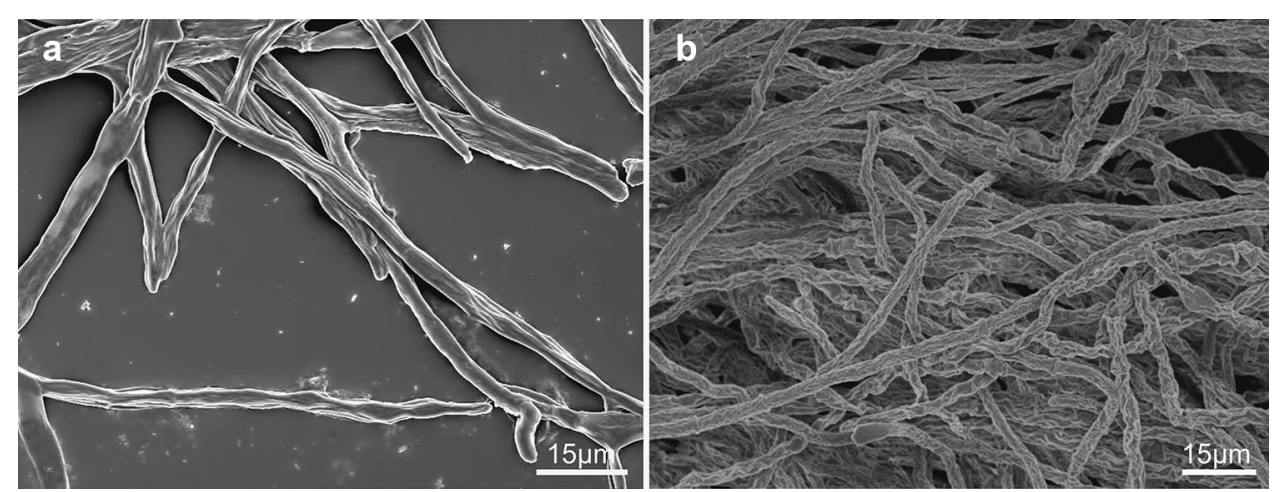


\section{Effect on protein content, SOD, and CAT}

Compared to the control group, the protein content of Fusarium oxysporum decreased until $18 \mathrm{~h}$ after exposure to the azoxystrobin nanosuspension (Fig. 12a). During the first $6 \mathrm{~h}$ of azoxystrobin nanosuspension exposure, the protein content increased. As a result of that, an oxidative stress response was generated and induced corresponding protein (Han et al. 2013; Hernández-Gea et al. 2013; Fucho et al. 2017). SOD enzymes, such as Mn-SOD in mitochondria and CuZn-SOD in cytosol, can eliminate superoxide anion-free radicals and protect organisms from oxidative damage (Mansuroğlu et al. 2015). As shown in Fig. 12b, the total SOD activity of the exposed group was less than that of the control group after $24 \mathrm{~h}$. The decreasing SOD activity suggested that the defense system of Fusarium oxysporum was compromised. Azoxystrobin was a wellknown mitochondrial respiration inhibitor. Therefore, the activity of Mn-SOD was investigated. Compared to that of the control group, a significant reduction in Mn-SOD activity was detected in azoxystrobin nanosuspension treatment (Fig. 12c). As seen from Fig. 12b, c, total SOD was principally derived from $\mathrm{CuZn}$-SOD in the cytosol. CAT was a vital enzyme in antioxidant systems as protecting organisms from ROS oxidative damage. The scavenge of $\mathrm{H}_{2} \mathrm{O}_{2}$ was mainly catalyzed by CAT(Kaneko and Ishii 2009). The CAT activity of the exposed group was approximately 2.53 -fold higher than that of the control group after $1 \mathrm{~h}$ (Fig. 12d) and then gradually decreased over time. These results implicated that $\mathrm{H}_{2} \mathrm{O}_{2}$ was enriched in fungi of the exposed group and led to oxidative damage. With prolonged exposure time, the decreasing activities of SOD and CAT indicated that the azoxystrobin nanosuspension could reduce the antioxidant defenses of Fusarium oxysporum.

\section{Conclusions}

For scale-up manufacture without organic residues, wet media milling technique has become more prevalent to enhance bioavailability of poorly soluble substance. In this study, the anionic surfactant SDS and polymeric PVP K30 were optimized from six conventional surfactants for the preparation of azoxystrobin nanosuspensions by wet media milling. The average diameter of the nanosuspension was approximately $200 \mathrm{~nm}$ in the dried state and the $\zeta$ potential was $-31.8 \mathrm{mV}$. Compared to WDG-A and WDG-B, the nanosuspension possessed the greater retention and the
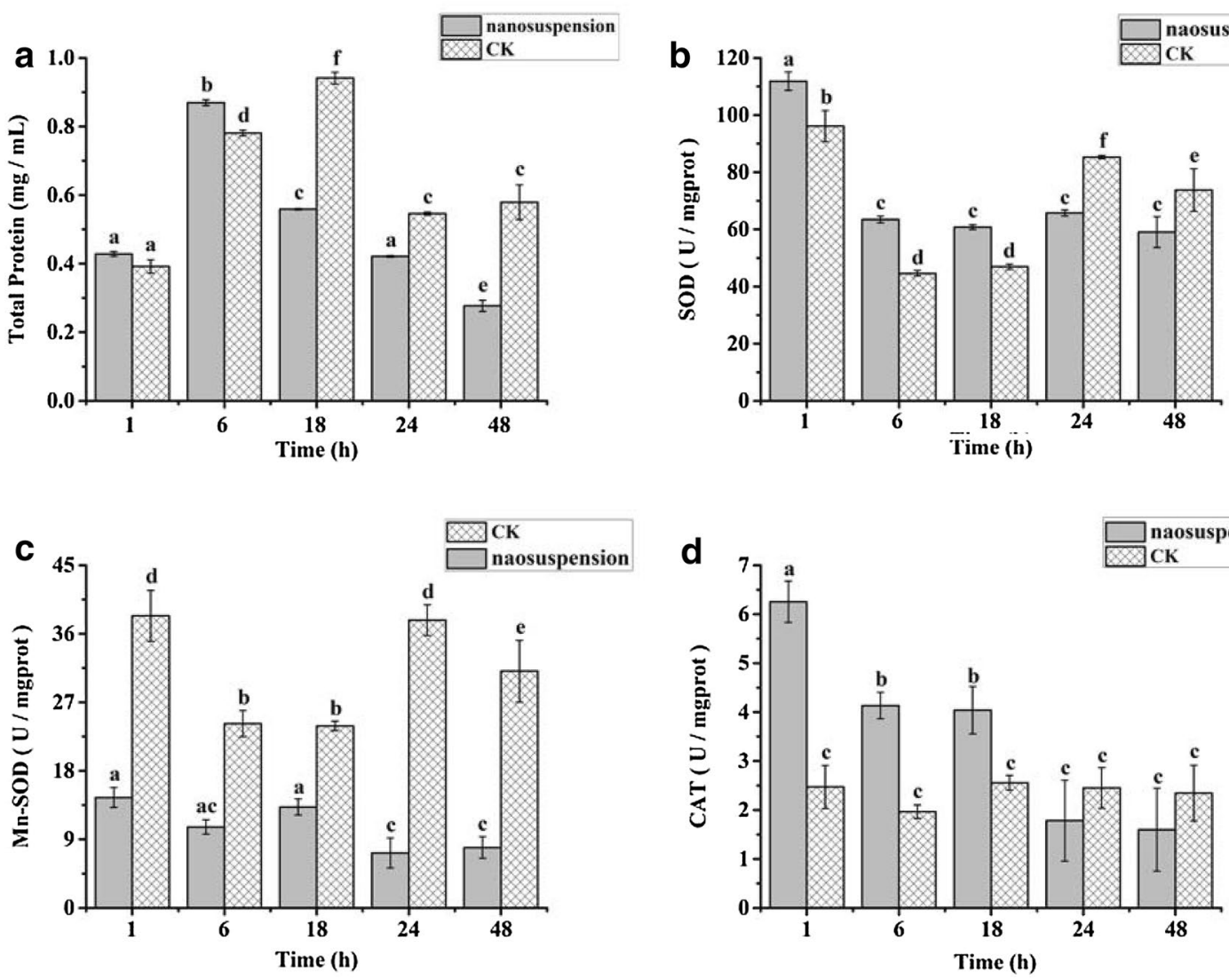

Fig. 12 Effect of the azoxystrobin nanosuspension on total protein content and anti-oxidase activities. a total protein; b SOD; c Mn-SOD; d CAT 
smaller contact angle on hydrophobic cabbage and hydrophilic cucumber leaves. Furthermore, the toxicity index of the azoxystrobin nanosuspension was approximately 1.7 -fold that of the other formulations against Fusarium oxysporum. Morphological alterations of Fusarium oxysporum were observed by optical microscopy and SEM. The hyphal deformations disclosed that the azoxystrobin nanosuspension can disturb cell walls and enhance cell wall permeability. Antioxidant enzyme activities were affected by the azoxystrobin nanosuspension and Fusarium oxysporum was more susceptible to oxidative damage. In conclusion, these findings reveal that the nanosuspension produced by wet media milling is a desirable nanoformulation for azoxystrobin to improve the antifungal activity.

Acknowledgements This study was financially supported by the Major National Scientific Research Program of China (No. 2014CB932200), the National Key Research and Development Program of China (2017YFD0201207, 2016YFD0200502), and the Agricultural Science and Technology Innovation Program (CAAS-XTCX2016004).

\section{References}

Ali HS, York P, Blagden N (2009) Preparation of hydrocortisone nanosuspension through a bottom-up nanoprecipitation technique using microfluidic reactors. Int J Pharm 375(1-2):107-113

Arciniegas-Grijalba PA, Patiño-Portela MC, Mosquera-Sánchez LP, Guerrero-Vargas JA, Rodríguez-Páez JE (2017) ZnO nanoparticles (ZnO-NPs) and their antifungal activity against coffee fungus erythricium salmonicolor. Appl Nanosci 7(5):225-241

Azevedo M-M, Carvalho A, Pascoal C, Rodrigues F, Cássio F (2007) Responses of antioxidant defenses to $\mathrm{Cu}$ and $\mathrm{Zn}$ stress in two aquatic fungi. Sci Total Environ 377(2):233-243

Bai YB, Zhang AL, Tang JJ, Gao JM (2013) Synthesis and antifungal activity of 2-chloromethyl-1 h-benzimidazole derivatives against phytopathogenic fungi in vitro. J Agric Food Chem 61(11):2789-2795

Brough C, Williams RO (2013) Amorphous solid dispersions and nanocrystal technologies for poorly water-soluble drug delivery. Int J Pharm 453(1):157-166

Castro T, Roggia S, Wekesa VW, de Andrade Moral R, Gb Demétrio C, Delalibera I, Klingen I (2016) The effect of synthetic pesticides and sulfur used in conventional and organically grown strawberry and soybean on neozygites floridana, a natural enemy of spider mites. Pest Manage Sci 72(9):1752-1757

Cho MS, Park SY, Hwang JY, Choi HJ (2004) Synthesis and electrical properties of polymer composites with polyaniline nanoparticles. Mater Sci Eng 24(1):15-18

Elsayed I, Abdelbary AA, Elshafeey AH (2014) Nanosizing of a poorly soluble drug: technique optimization, factorial analysis, and pharmacokinetic study in healthy human volunteers. Int J Nanomed 9:2943-2953

Fucho R, Vallejo C, Alarcon-Vila C, Garcia-Ruiz C, Fernandez-Checa J (2017) Thu-326-ethanol feeding preferentially increases steroidogenic acute regulatory protein, mitochondrial respiration and oxidative stress in perivenous mouse hepatocytes. J Hepatol 66(1):S115

Gao X, Wen X, Yu CA, Esser L, Tsao S, Quinn B, Zhang L, Yu L, Xia D (2002) The crystal structure of mitochondrial cytochrome bc1 in complex with famoxadone: the role of aromatic-aromatic interaction in inhibition. Biochemistry 41(39):11692-11702

George M, Ghosh I (2013) Identifying the correlation between drug/ stabilizer properties and critical quality attributes (CQAs) of nanosuspension formulation prepared by wet media milling technology. Eur J Pharm Sci 48(1):142-152

Ghosh RK, Singh N (2009) Leaching behaviour of azoxystrobin and metabolites in soil columns. Pest Manage Sci 65(9):1009-1014

Ghosh I, Schenck D, Bose S, Ruegger C (2012) Optimization of formulation and process parameters for the production of nanosuspension by wet media milling technique: effect of vitamin E TPGS and nanocrystal particle size on oral absorption. Eur J Pharm Sci 47(4):718-728

Han J, Back SH, Hur J, Lin Y-H, Gildersleeve R, Shan J, Yuan CL, Krokowski D, Wang S, Hatzoglou M (2013) ER-stress-induced transcriptional regulation increases protein synthesis leading to cell death. Nat Cell Biol 15(5):481-490

Hernández-Gea V, Hilscher M, Rozenfeld R, Lim MP, Nieto N, Werner S, Devi LA, Friedman SL (2013) Endoplasmic reticulum stress induces fibrogenic activity in hepatic stellate cells through autophagy. J Hepatol 59(1):98-104

Holzapfel V, Musyanovych A, Landfester K, Lorenz MR, Mailander V (2005) Preparation of fluorescent carboxyl and amino functionalized polystyrene particles by miniemulsion polymerization as markers for cells. Macromol Chem Phys 206(24):2440-2449

Ibrahim N, Ibrahim H, Dormoi J, Briolant S, Pradines B, Moreno A, Mazier D, Legrand P, Nepveu F (2014) Albumin-bound nanoparticles of practically water-insoluble antimalarial lead greatly enhance its efficacy. Int J Pharm 464(1):214-224

Inoue K, Tsurumi T, Ishii H, Park P, Ikeda K (2011) Cytological evaluation of the effect of azoxystrobin and alternative oxidase inhibitors in botrytis cinerea. FEMS Microbiol Lett 326(1):83-90

Ito A, Konnerth C, Schmidt J, Peukert W (2016) Effect of polymer species and concentration on the production of mefenamic acid nanoparticles by media milling. Eur J Pharm Biopharm 98:98-107

Kah M, Hofmann T (2014) Nanopesticide research: current trends and future priorities. Environ Int 63:224-235

Kaneko I, Ishii H (2009) Effect of azoxystrobin on activities of antioxidant enzymes and alternative oxidase in wheat head blight pathogens fusarium graminearum and microdochium nivale. $\mathbf{J}$ Gen Plant Pathol 75(5):388-398

Kargosha K, Shirazi Z (2015) Determination of water content of crystalline pharmaceutical solids under different percentages of relative humidity. Pharm Sci 21(3):127-135

Krupa A, Descamps M, Willart JF, Strach B, Wyska E, Jachowicz R, Danede F (2016) High-energy ball milling as green process to vitrify tadalafil and improve bioavailability. Mol Pharm 13(11):3891-3902

Kumar Singh S, Vaidya Y, Gulati M, Bhattacharya S, Garg V, Kumar Pandey N (2016) Nanosuspension: principles, perspectives and practices. Curr Drug Deliv 13(8):1222-1246

Kumari A, Kumar J, Shakil N, Kamil D (2015) Bio-efficacy evaluation of cr formulations of azoxystrobin against rhizoctonia solani. Ann Plant Prot Sci 23(1):124-126

Lee D, Lee BC, Park KH, Ryu HJ, Jeon S, Hong SH (2015) Scalable exfoliation process for highly soluble boron nitride nanoplatelets by hydroxide-assisted ball milling. Nano Lett 15(2):1238-1244

Leng D, Chen H, Li G, Guo M, Zhu Z, Xu L, Wang Y (2014) Development and comparison of intramuscularly long-acting paliperidone palmitate nanosuspensions with different particle size. Int J Pharm 472(1):380-385

Li M, Azad MAK, Dave RN, Bilgili E (2016) Nanomilling of drugs for bioavailability enhancement: a holistic formulation-process perspective. Pharmaceutics 8(2):17 
Lin H, Zhou H, Xu L, Zhu H, Huang H (2016) Effect of surfactant concentration on the spreading properties of pesticide droplets on eucalyptus leaves. Biosyst Eng 143:42-49

Liu L, Zhu B, Wang G-X (2015) Azoxystrobin-induced excessive reactive oxygen species (ROS) production and inhibition of photosynthesis in the unicellular green algae chlorella vulgaris. Environ Sci Pollut Res 22(10):7766-7775

Mansuroğlu B, Derman S, Yaba A, Kızılbey K (2015) Protective effect of chemically modified sod on lipid peroxidation and antioxidant status in diabetic rats. Int J Biol Macromol 72:79-87

Mcallister TD, Farrand LD, Howdle SM (2016) Improved particle size control for the dispersion polymerization of methyl methacrylate in supercritical carbon dioxide. Macromol Chem Phys 217(20):2294-2301

Mirza RM (2017) A nanocrystal technology: to enhance solubility of poorly water soluble drugs. J Appl Pharm Res 5(1):1-13

Moerz ST, Huber P (2015) Protein adsorption into mesopores: a combination of electrostatic interaction, counterion release and van der Waals Forces. Langmuir 30(10):2729-2737

Murdande SB, Shah DA, Dave RH (2015) Impact of nanosizing on solubility and dissolution rate of poorly soluble pharmaceuticals. J Pharm Sci 104(6):2094-2102

Ninjbadgar T, Fox E, Hierrezuelo J, Haddassi FE, Brougham DF (2015) Monodisperse magnetic nanoparticle assemblies prepared at scale by competitive stabiliser desorption. J Mater Chem B 3(44):8638-8643

Olsvik PA, Kroglund F, Finstad B, Kristensen T (2010) Effects of the fungicide azoxystrobin on atlantic salmon (salmo salar 1.) smolt. Ecotoxicol Environ Saf 73(8):1852-1861

Palermo RN, Anderson CA, Drennen JK (2012) Review: use of thermal, diffraction, and vibrational analytical methods to determine mechanisms of solid dispersion stability. J Pharm Innov 7(1):2-12

Pamies R, Cifre JGH, Espin VF, Colladogonzalez M, Banos FGD, La Torre JGD (2014) Aggregation behaviour of gold nanoparticles in saline aqueous media. J Nanoparticle Res 16(4):2376

Qin CF, He MH, Chen FP, Zhu W, Yang LN, Wu EJ, Guo ZL, Shang LP, Zhan J (2016) Comparative analyses of fungicide sensitivity and SSR marker variations indicate a low risk of developing azoxystrobin resistance in phytophthora infestans. Sci Rep 6:20483

Radović T, Grujić S, Petković A, Dimkić M, Laušević M (2015) Determination of pharmaceuticals and pesticides in river sediments and corresponding surface and ground water in the Danube River and tributaries in Serbia. Environ Monit Assess 187(1):4092

Rance GA, Marsh DH, Bourne SJ, Reade TJ, Khlobystov AN (2010) Van der waals interactions between nanotubes and nanoparticles for controlled assembly of composite nanostructures. ACS Nano 4(8):4920-4928
Rodrigues ET, Lopes I, Pardal MA (2013) Occurrence, fate and effects of azoxystrobin in aquatic ecosystems: a review. Environ Int 53:18-28

Singh B, Jang Y, Maharjan S, Kim HJ, Lee AY, Kim S, Gankhuyag N, Yang MS, Choi YJ, Cho MH (2017) Combination therapy with doxorubicin-loaded galactosylated poly (ethyleneglycol)-lithocholic acid to suppress the tumor growth in an orthotopic mouse model of liver cancer. Biomaterials 116:130-144

Sun Y, Mei Y, Quan J, Xiao X, Zhang L, Tian D, Li H (2016) The macroscopic wettable surface: fabricated by calix [4] arene-based host-guest interaction and chiral discrimination of glucose. Chem Commun 52(100): 14416-14418

Symonds B, Lindsay CI, Thomson NR, Khutoryanskiy VV (2016) Chitosan as a rainfastness adjuvant for agrochemicals. RSC Adv 6(104):102206-102213

Taylor LS, Zhang GG (2016) Physical chemistry of supersaturated solutions and implications for oral absorption. Adv Drug Deliv Rev 101:122-142

Teeranachaideekul V, Junyaprasert VB, Souto EB, Müller RH (2008) Development of ascorbyl palmitate nanocrystals applying the nanosuspension technology. Int J Pharm 354(1):227-234

Turrens JF, Boveris A (1980) Generation of superoxide anion by the NADH dehydrogenase of bovine heart mitochondria. Biochem J 191(2):421-427

Verma A, Uzun O, Hu Y, Hu Y, Han H, Watson N, Chen S, Irvine DJ, Stellacci F (2008) Surface-structure-regulated cell-membrane penetration by monolayer-protected nanoparticles. Nat Mater 7(7):588-595

Yadollahi R, Vasilev K, Simovic S (2015) Nanosuspension technologies for delivery of poorly soluble drugs. J Nanomater 2015:1-13

Yue P, Xiao M, Xie Y, Ma Y, Guan Y, Wu Z, Hu P, Wang Y (2015) The roles of vitrification of stabilizers/matrix formers for the redispersibility of drug nanocrystals after solidification: a case study. AAPS Pharm 17(6):1274-1284

Zeng X, Ma J, Luo L, Yang L, Cao X, Tian D, Li H (2015) Pesticide macroscopic recognition by a naphthol-appended calix[4]arene. Org Lett 17(12):2976-2979

Zhong Q, Jin M (2009) Zein nanoparticles produced by liquid-liquid dispersion. Food Hydrocoll 23(8):2380-2387

Publisher note Springer Nature remains neutral with regard to jurisdictional claims in published maps and institutional affiliations. 